\section{Un implant bioactif pour prévenir la maladie d'Alzheimer}

Aurélien Lathuilière ${ }^{1}$, Bernard L. Schneider ${ }^{2}$
${ }^{1}$ Service de neurologie, Hôpitaux Universitaires de Genève, rue Gabrielle-Perret-Gentil 4, CH-1211 Genève, Suisse.

${ }^{2}$ Brain and Mind Institute, École Polytechnique Fédérale de Lausanne (EPFL), CH-1015 Lausanne, Suisse. aurelien.lathuiliere@hcuge.ch bernard.schneider@epfl.ch
> Les maladies neurodégénératives représentent un défi de santé majeur pour les sociétés dont l'espérance de vie a augmenté durant les dernières décennies. L'une d'entre elles, la maladie d'Alzheimer, est la cause la plus fréquente de démence. Elle provoque des troubles cognitifs dont l'évolution mène à une perte d'autonomie progressive et à une altération dramatique de la qualité de vie des patients et de leurs aidants. Les traitements actuellement disponibles permettent de traiter partiellement ces symptômes, sans pouvoir modifier la progression de la maladie.

Les connaissances des mécanismes physiopathologiques menant à la dégénérescence des neurones ont remarquablement progressé. Le dépôt de formes agrégées du peptide amyloïde bêta $(A \beta)$ est l'une des manifestations de la maladie d'Alzheimer, survenant à un stade très précoce dans le processus pathogénique. Ces dépôts apparaissent sous la forme de plaques extracellulaires visibles dans certaines régions du tissu cérébral. La pathologie amyloïde est suivie d'autres événements pathologiques, tels que la formation de dégénérescences neurofibrillaires à l'intérieur des neurones. Il s'agit d'enchevêtrements de fibrilles composées principalement de la protéine tau (tubule-associated unit) hyperphosphorylée. Ce n'est que récemment que l'identification de biomarqueurs par imagerie moléculaire ${ }^{1}$ ou morpho-

${ }^{1}$ L'imagerie moléculaire est fondée sur l'utilisation d'agents de contraste spécifiques contenant un composé qui cible une molécule particulière : enzyme, protéine ou récepteur de membrane qui vont être le reflet de l'activation ou de l'altération d'une voie de signalisation cellulaire. logique ${ }^{2}$, ou par analyses biochimiques dans le liquide céphalo-rachidien, a permis de suivre ces signes de la maladie au cours du temps. Ces processus pathologiques sont très progressifs, et la formation de plaques amyloïdes débute des années, voire des décennies, avant que les premiers symptômes de la maladie ne se manifestent. Par conséquent, des stratégies thérapeutiques ciblées visant à interférer avec l'accumulation d'A $\beta$ ou de la protéine tau ont été proposées, dans l'espoir de freiner l'évolution de la maladie $[1,2](\rightarrow)$.

$(\rightarrow)$ Voir les Nouvelles de G. Dorothée et P. Aucouturier, $m / s n^{\circ} 11$, novembre 2011, page 938, et de L. Ozmen et L. Collin, $\mathrm{m} / \mathrm{s} \mathrm{n}^{\circ} 2$, février 2015

\section{Stratégie}

thérapeutique page 132

\section{par immunisation}

L'approche thérapeutique actuellement la plus avancée est basée sur l'immunisation contre $A \beta$, soit par vaccination, soit par administration directe d'anticorps monoclonaux dirigés contre des formes pathologiques de ce peptide [3]. De nombreuses études réalisées dans des modèles de souris transgéniques de la maladie d'Alzheimer ont démontré que la vaccination ou l'administration d'anticorps dirigés contre le peptide $A \beta$ peuvent empêcher la formation de dépôts amyloïdes, avec des effets protecteurs sur les mécanismes moléculaires qui découlent de cette pathologie et qui mènent ultimement à la neurodé-

L'imagerie morphologique regroupe par exemple la radiographie, le scanner, l'échographie, l'imagerie par résonance magnétique (IRM). Ce sont des imageries qui prennent une photographie de l'organisme sans en étudier le fonctionnement. générescence (voir l'étude princeps [4]). Ces résultats ont motivé le lancement de plusieurs essais cliniques avec des patients atteints de la maladie d'Alzheimer, allant jusqu'en phase III. Les résultats de ces études ont pour la plupart été décevants. Néanmoins, les essais cliniques les plus récents, utilisant des anticorps dirigés contre certaines formes d'A $\beta$ plus spécifiquement associées à la pathologie (par exemple l'Aducanumab, le Gantenerumab, le Crenezumab ou le Solanezumab), ont montré des effets prometteurs. La sélection de patients à des stades précoces de la maladie, sur la base de biomarqueurs, et l'administration d'anticorps à fortes doses (> $5 \mathrm{mg} / \mathrm{kg}$ ), semblent être des éléments importants permettant de potentialiser les effets du traitement. Certains de ces anticorps non seulement diminuent le nombre de plaques amylö̈des, mais semblent également ralentir le déclin des facultés cognitives, un résultat qui reste néanmoins à confirmer par des essais de phase III $[5,6]$. Toutefois, ces traitements ne sont pas dénués d'effets secondaires. En effet, chez certains sujets traités, des anomalies nommées ARIA (amyloid related imaging abnormalities) sont décrites, détectables en imagerie par résonance magnétique de suivi, soit sous forme de microsaignements, soit sous forme d'œdèmes vasogéniques ${ }^{3}$ [7]. Ces anomalies apparaissent plus fréquemment chez les patients recevant les doses les plus élevées d'anticorps.

\footnotetext{
3 Fedèmes cérébraux causés par la rupture des jonctions serrées des cellules de la membrane endothéliale formant la barrière hémato-encéphalique.
} 


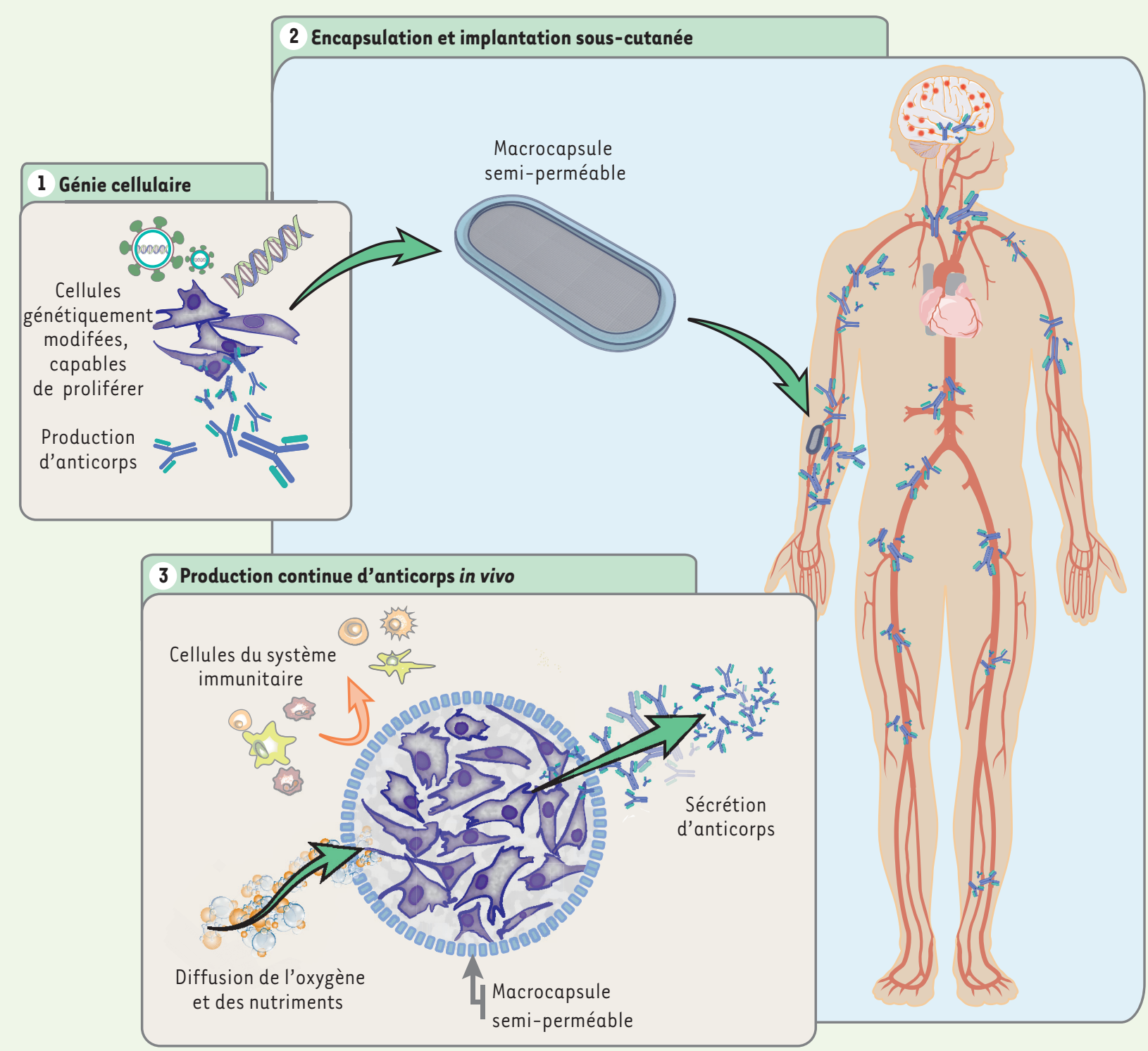

Figure 1. Vue schématique de l'administration sous-cutanée d'anticorps par la technique d'encapsulation cellulaire. 1. Une lignée cellulaire plateforme est modifiée génétiquement pour la sécrétion de l'anticorps d'intérêt. 2. Ces cellules modifiées sont introduites à l'intérieur d'un dispositif d'encapsulation composé d'une membrane semi-perméable. Ce dispositif est implanté dans le tissu sous-cutané. 3. La membrane semi-perméable isole les cellules génétiquement modifiées des cellules du système immunitaire de l'hôte. La membrane permet la diffusion passive d'oxygène et de nutriments vers l'intérieur du dispositif, alors que les anticorps recombinants produits par les cellules sont continuellement sécrétés par la capsule.

Elles sont parfois associées à une aggravation des symptômes neurologiques, ce qui peut entraîner l'interruption du traitement.

Bien qu'encourageantes, les données acquises lors de ces essais cliniques mettent en exergue certaines limitations du traitement, qu'il conviendra d'appréhender pour tirer un bénéfice maximal de cette stratégie thérapeutique. Pour obtenir un effet sur le fonctionnement cognitif des patients, il apparaît nécessaire d'intervenir très tôt dans le cours de la maladie, de préférence à un stade de trouble cognitif léger, voire même pré-symptomatique, afin de pouvoir prévenir les dommages généralement irréversibles du tissu cérébral. Dans ce but, les patients devraient se soumettre à intervalle régulier à des administrations d'anticorps (typiquement chaque mois), et ce pendant plusieurs années. L'ajustement du dosage représente cependant un défi, les doses faibles n'ayant pas montré d'effet alors que les doses élevées induisent fréquemment des effets secondaires. 
Il existe donc une nécessité de développer de nouveaux modes d'administration d'anticorps monoclonaux, mieux adaptés au traitement de processus pathologiques intracérébraux chroniques.

\section{Immunisation utilisant la technologie} d'encapsulation cellulaire

Une option thérapeutique consiste à développer des méthodes biotechnologiques pour administrer de manière continue les anticorps, afin d'éviter les pics de dose pouvant entraîner des effets secondaires. Une stratégie proposée est fondée sur la technologie d'encapsulation cellulaire. Cette technique utilise un dispositif implantable, composé d'une membrane de polymères semi-perméable, contenant des cellules issues d'une lignée génétiquement modifiée dans le but de produire une protéine thérapeutique (méthodologie décrite dans [8]). La fonction de la membrane est de protéger les cellules implantées contre l'immunité cellulaire de l'hôte, ce qui permet d'utiliser pour tous les receveurs une lignée cellulaire unique, renouvelable et bien caractérisée, sans qu'aucun traitement immunosuppresseur ne soit nécessaire. Cette membrane est suffisamment perméable pour que les échanges d'oxygène et de nutriments garantissent la survie des cellules greffées tout en laissant diffuser passivement la protéine recombinante qu'elles produisent, par exemple un anticorps à usage thérapeutique. En outre, le volume du dispositif définit sa contenance en cellules, ce qui permet d'évaluer la dose d'anticorps produite de manière chronique. Dans le cas où le traitement devrait être interrompu, le dispositif peut être explanté par une intervention chirurgicale bénigne. Cette technologie est illustrée dans la Figure 1. Nous avons conçu un dispositif d'encapsulation cellulaire pour l'implantation de plusieurs millions de cellules dans le tissu sous-cutané. Les caractéristiques intrinsèques innovantes de cet implant, associées à l'utilisation d'une matrice extracellulaire artificielle biodégra- dable, ont permis la survie de cellules allogéniques transplantées durant plus d'une année dans un modèle animal [9]. Avec l'optimisation des méthodes de modification génétique, il est possible de générer des lignées de myoblastes $\mathrm{C} 2 \mathrm{C} 12^{4}$ capables de survivre dans notre dispositif, tout en produisant des anticorps recombinants à un taux élevé, atteignant $100 \mu \mathrm{g}$ par jour pour une capsule adaptée à la souris [10]. L'objectif suivant a été de démontrer l'efficacité thérapeutique de cette approche pour l'administration continue d'anticorps dirigés contre le peptide $A \beta$ dans un modèle animal de la maladie d'Alzheimer.

Nous avons généré une lignée de myoblastes murins $\mathrm{C} 2 \mathrm{Cl} 2$ génétiquement modifiés pour la production d'un anticorps de type immunoglobuline G (IgG) dirigé spécifiquement contre une forme agrégée du peptide $A \beta$ [9]. L'anticorps produit possède des caractéristiques très proches du Gantenerumab, un anticorps actuellement testé en essai clinique. Ces cellules ont été intégrées au dispositif d'encapsulation décrit cidessus, puis implantées dans le tissu sous-cutané de deux types de souris transgéniques. Les souris $5 X F A D^{5}$ développent des dépôts de plaques amyloïdes dès l'âge de 2 mois, tandis que les souris TauPS2APP présentent une déposition d'A $\beta$ plus progressive, mais associée à une pathologie de type neurofibrillaire caractérisée par une hyper-phosphorylation de la protéine tau. Dans les deux modèles, l'implantation sous-cutanée de cellules encapsulées a pu être utilisée avec succès pour l'administration continue d'anticorps, détectés dans le plasma à des taux stables excédant $50 \mu \mathrm{g} / \mathrm{ml}$ pendant près de 10 mois. Ces anticorps circulants sont capables d'atteindre le cerveau des souris traitées et de se lier aux plaques amyloïdes. La présence de dépôts amyloïdes dans le

\footnotetext{
${ }^{4}$ La lignée cellulaire $\mathrm{C} 2 \mathrm{C} 12$ de myoblastes a été isolée à partir du muscle de souris $\mathrm{C} 3 \mathrm{H}$.

${ }^{5}$ Les souris 5 XFAD et TauPS2APP représentent des modèles transgéniques de maladie d'Alzheimer.
}

cortex a été quantifiée par immunohistochimie et biochimie, ainsi que par imagerie tomographique aux rayons $X$ avec contraste de phase. Ces analyses ont montré que le traitement est capable de diminuer drastiquement la formation de plaques amyloïdes, en particulier lorsque la capsule produisant les anticorps anti-A $\beta$ est implantée avant la formation des premiers dépôts. De plus, dans les souris TauPS2APP, cette réduction de la pathologie amyloïde s'accompagne d'une diminution significative de la présence pathologique de formes hyper-phosphorylées de la protéine tau dans l'hippocampe. Concernant l'origine de ces effets, nous avons noté chez les souris traitées une augmentation du nombre de cellules microgliales autour des plaques amyloïdes. Cette observation laisse penser que l'anticorps lié aux plaques pourrait faciliter la phagocytose et la dégradation des dépôts amyloïdes par la microglie, ce que confirment des expériences conduites in vitro [11, 12].

\section{Conclusions}

Ces résultats confirment l'efficacité de l'immunisation passive par anticorps monoclonaux dirigés contre I'A $\beta$ pour la prévention de la maladie d'Alzheimer. De plus, ils valident I'utilisation de la technologie d'encapsulation cellulaire comme moyen d'administration continu d'anticorps thérapeutiques par voie sous-cutanée. Les anticorps produits en périphérie peuvent atteindre le tissu cérébral en dépit de la présence de la barrière hémato-encéphalique qui constitue un obstacle à la diffusion. Dans le cas de pathologies pour lesquelles le seuil thérapeutique serait plus élevé et de plus grandes quantités d'anticorps devraient atteindre le tissu cérébral, il serait possible d'utiliser un dispositif similaire permettant d'implanter des cellules productrices d'anticorps directement dans le système nerveux central.

Grâce à la très haute spécificité des anticorps monoclonaux, il est aujourd'hui possible d'interférer avec 
des processus pathogéniques complexes. Cette technologie, associée à la grande adaptabilité de l'encapsulation cellulaire pour l'administration de protéines recombinantes, permet d'envisager de nouvelles options thérapeutiques qu'il convient d'investiguer non seulement en neurologie, mais aussi dans d'autres domaines de la médecine. Notre travail actuel vise donc à transférer cette technologie vers une application clinique. $\diamond$

A bioactive implantable device to prevent Alzheimer's disease

\section{LIENS D'INTÉRÊT}

Les auteurs déclarent n'avoir aucun lien d'intérêt concernant les données publiées dans cet article.

\section{RÉFÉRENCES}

1. Dorothée $G$, Aucouturier P. Les réponses immunitaires adaptatives contre le peptide amyloïde $A \beta$ dans la maladie d'Alzheimer : leçons et perspectives. Med Sci (Paris) $2011 ; 27:$ 938-40.

2. Ozmen L, Collin L. Prévenir la progression de la pathologie Tau par immunothérapie passive. Med Sci (Paris) $2015 ; 31: 132-4$.

3. Wisniewski T, Goni F. Immunotherapeutic approaches for Alzheimer's disease. Neuron $2015 ; 85$ : 1162-76.

4. Schenk D, Barbour R, Dunn W, et al. Immunization with amyloid-beta attenuates Alzheimer-disease-like pathology in the PDAPP mouse. Nature $1999 ; 400$ : $173-7$.

5. Ostrowitzki S, Deptula D, Thurfjell L, et al. Mechanism of amyloid removal in patients with Alzheimer disease treated with gantenerumab. Arch Neurol 2012 ; 69 : 198-207.

6. Reardon S. Antibody drugs for Alzheimer's show glimmers of promise. Nature $2015 ; 523: 509-10$.

7. Sperling RA, Jack CR Jr, Black SE, et al. Amyloidrelated imaging abnormalities in amyloid-modifying therapeutic trials: recommendations from the Alzheimer's association research roundtable workgroup. Alzheimers Dement 2011 ; 7 : 367-85.

8. Lathuiliere A, Mach N, Schneider BL. Encapsulated cellular implants for recombinant protein delivery and therapeutic modulation of the immune system. Int J Mol Sci $2015 ; 16$ : 10578-600.

9. Lathuiliere A, Cosson S, Lutolf MP, et al. A highcapacity cell macroencapsulation system supporting the long-term survival of genetically engineered allogeneic cells. Biomaterials 2014 ; 35 : 779-91.

10. Lathuiliere A, Bohrmann B, Kopetzki $\varepsilon$, et al. Genetic engineering of cell lines using lentiviral vectors to achieve antibody secretion following encapsulated implantation. Biomaterials 2014 ; 35 : 792-802.

11. Lathuiliere A, Laversenne V, Astolfo A, et al. A subcutaneous cellular implant for passive immunization against amyloid-beta reduces brain amyloid and tau pathologies. Brain 2016 ; 139 : 1587 604

12. Xiang $X$, Werner $G$, Bohrmann B, et al. TREM2 deficiency reduces the efficacy of immunotherapeutic amyloid clearance. EMBO Mol Med 2016 ; 8 : 992-1004.

\section{NOUVELL\&}

Un atlas du potentiel
neuroplastique chez les
patients cérébrolésés
Guillaume Herbet $^{1-3}$, Gilles Lafargue ${ }^{4}$, Hugues Duffau

> Le système nerveux central humain peut être affecté par des processus pathologiques variés causant, la plupart du temps, des brèches plus ou moins étendues dans l'architecture neurale. Ces lésions cérébrales sont soit aiguës (c'est-à-dire se produisant de façon brutale), comme dans le cas de l'accident vasculaire ischémique, soit progressives et diffuses, comme dans le cas des maladies neurodégénératives (par exemple la maladie d'Alzheimer) ou des tumeurs neurologiques à progression lente (par exemple les gliomes diffus de bas grade). Dans ces deux cas de figure, le cerveau humain est confronté à un défi majeur: préserver son architecture fonctionnelle malgré les dommages structuraux induits par la lésion.

Récemment encore, il était généralement admis par la communauté scientifique que le potentiel neuroplastique du cerveau, c'est-à-dire sa capacité à se réorganiser lui-même en réponse à un événement pathologique, était très limité. Cette croyance s'appuyait avant tout sur les observations réalisées chez les patients atteints d'un accident vasculaire cérébral. Le plus souvent, ces patients souffrent de déficits fonctionnels durables, difficilement compensables, malgré la mise en place de stratégies intensives de réhabilitation fonctionnelle. Ceci a grandement contribué à conforter l'idée - fausse - selon laquelle le cerveau humain se caractérise par une organisation neurale figée. Cette idée, enracinée dans la vieille thèse du localisationnisme ${ }^{l}$ cortical du $\mathrm{XIX}$ e siècle, qui dépeint le cerveau comme

${ }^{1}$ Théorie attribuant une fonction cérébrale à une aire précise du cerveau.
${ }^{1}$ Département de neurochirurgie, Hôpital Gui de Chauliac, CHU de Montpellier, 80, avenue Augustin Fliche, 34295 Montpellier, France ;

2 Inserm, U-1051, équipe 4, Institut des Neurosciences de Montpellier, F-34091 Montpellier, France ;

${ }^{3}$ Université de Montpellier, F-34000

Montpellier, France ;

${ }^{4}$ Université de Lille, EA 4072 - PSITEC

(Psychologie, interactions, temps, émotions, cognition), F-59000 Lille, France.

guillaume.herbet@gmail.com

une vaste mosaïque de structures hyperspécialisées et donc non interchangeables d'un point de vue fonctionnel, est encore omniprésente aujourd'hui. Selon la logique de ce courant de pensée, une lésion d'une structure fonctionnelle induit nécessairement une perturbation comportementale ou cognitive spécifique et, surtout, irréversible. Pourtant, en parallèle, des cas d'atteinte massive du système nerveux central, sans conséquence fonctionnelle durable, ont été régulièrement décrits dans la littérature scientifique et médicale, mettant ainsi à mal la valeur heuristique de ce modèle de fonctionnement. Le système nerveux central a donc la capacité, au moins dans certaines circonstances, de compenser des circuits neuronaux endom- 